\title{
Comparing the formation of lutein nanodispersion prepared by using solvent displacement method and high-pressure valve homogenization: effects of formulation parameters
}

\begin{abstract}
In this study, we compared the effect of formulation parameters on the physicochemical properties of lutein nanodispersions prepared using a low- and high-energy approach, i.e., solvent displacement (SD) and high-pressure valve homogenization (HPH), respectively. The lutein concentration had a significant effect on the particle size (PS) and particle size distribution (PSD) of nanodispersions that were prepared by using both methods. However, Tween 80 concentration and organic-phase-to-aqueous-phase volume ratio (OAR) only had a significant effect on the PS of nanodispersions prepared by SD. Under all the variations in the formulation parameters, the PSs and PSDs of nanodispersions prepared by SD and HPH were not significantly different. At $0.1 \%$ lutein concentration, $0.1 \%$ Tween 80 concentration and OAR of 1:9, the nanodispersions prepared by using both methods displayed minimum PS and excellent lutein retentions $(>90 \%)$. This study showed that SD is a suitable alternative to HPH for preparing lutein nanodispersions.
\end{abstract}

Keyword: Nanodispersion; Solvent displacement; High-pressure homogenization; Comparison; Lutein 